\title{
Photophysical Processes Exploited in Digital Imaging Microscopy: Fluorescence Resonance Energy Transfer and Delayed Luminescence
}

\author{
T. M. Jovin, G. Marriott, R. M. Clegg, and D. J. Arndt-Jovin
}

Abteilung Molekulare Biologie, Max-Planck-Institut für Biophysikalische Chemie, Postfach 2841, D-3400 Göttingen, Federal Republic of Germany

\section{Energy Transfer / Fluorescence / Luminescence / Microscopy / Phosphorescence}

The spectroscopic techniques of fluorescence resonance energy transfer and of time-resolved delayed fluorescence and phosphorescence have been introduced into a microscope equipped with a solid-state CCD camera and phase-locked excitation and emission choppers. The distribution and replication of DNA in cells has been quantitated by these methods, as well as by confocal laser scanning microscopy Sites of replication in living cells can be uniquely identified by delayed luminescence.

\section{Introduction}

Cellular functions are mediated by complex interactions between small effector molecules and the major macromolecules-proteins, DNA, RNA-present as isolated or multicomponent structures. Thus, an elucidation of the mechanisms operative within and between cells requires a quantitative description of spatial and temporal molecular distributions extending over many orders of magnitude (e.g. nanometer$\mathrm{cm}, \mu \mathrm{s}-\mathrm{min})$. The light microscope is an essential instrument for such determinations, and the contrast required in image formation can be generated by numerous optical phenomena ([1], Table 1 in Ref. [2]). However, it is only since the advent of digital microscopes, integrating optical detectors with digital computers, that low light level luminescence has been applied quantitatively in studies of individual cells. In this report, we describe the use of photophysical processes that extend the resolution of the optical microscope beyond the limit dictated by diffraction. The latter still defines the practical size of individual pixels and voxels (the individual picture elements in two and three dimensions, respectively). However, one can introduce into the imaging process spectroscopic phenomena that operate over molecular dimensions and offer very high sensitivity, selectivity, and contrast. These include fluorescence resonance energy transfer for assessing molecular proximities in the nanometer range, and delayed emission potentiated by external heavy atoms located specifically in target structures, e.g. at sites of DNA replication in living cells labeled by incorporation of the base analog bromodeoxyuridine (BrdU). These approaches are being extended to the confocal laser scanning microscope in order to provide the additional benefits of high resolution optical sectioning and 3-D reconstruction.

Imaging by light emission involves the three sequential processes of illumination (excitation), emission, and detection. The structure, localization, orientation, and environment of the luminophore determine the relevant photophysical properties. In some of the measurements described below, the duration of the excited state relative to the period of observation is of central importance. For example, prompt fluorescence is generally characterized by nanosecond lifetimes and observed in the microscope as a steadystate signal, whereas the fluorescence decay of longer-lived singlets and the delayed luminescence from triplet states may be easily resolved in the temporal domain.

\section{Systems for Luminescence Digital Imaging Microscopy (LDIM)}

The majority of LDIM systems in current use incorporate TV cameras as detectors. However, such units generally demonstrate temporal and geometric instabilities, are noisy and limited in dynamic range, and have a non-linear response to light intensity. Slow-scan, high resolution cameras incorporating solid-state charge-coupled device (CCD) arrays, and laser scanning systems with photomultipler detectors are free from these problems. Thus, they are ideally suited for quantitative LDIM. Together with the continuously expanding array of specific luminescence probes, these systems constitute a chemical-technological "triad" driving the current explosive development in optical microscopy (Fig. 1, [3]).

Probes
-vital stains: macromolecules, ions
-monoclonal antibodies
-nucleic acid sequences
-estimators of quantitity, distance, mobility
-functional: growth, differentiation, malignancy

Probe and instrumentation technologies in contemporary luminescence digital imaging microscopy (LDIM). Their combination has led to new quantitative measurements of photophysical properties and cellular functions, and an extension of the resolution of the light microscope beyond the nominal diffraction limit

\subsection{CCD Array Cameras}

The basic characteristics of CCDs correspond to those of an almost ideal light detector: low dark noise in the case of thermoelectrically or cryogenically cooled devices, geometric accuracy and stability, linearity of response over a high dynamic range, and high quantum efficiency. Since noise is dominated by the readout electronics (output amplifier), the optical signal can be integrated until the potential wells of the individual pixels are filled to capacity with photoelectrons or for as long as desired in time sampled measurements. Readout speeds in CCD cameras are determined by the sensor and the associated electronic circuitry, and lie 
typically in the range of $50 \mathrm{KHz}-5 \mathrm{MHz}$ pixel rates. The recent developments in $\mathrm{CCD}$ technology have been reviewed extensively [4].

In the applications decribed below, images were acquired with a Photometrics (Tucson, Arizona) Series 200 camera system incorporating: (i) a mechanical shutter; (ii) a thermoelectrically cooled Thomson CSF Model TH7882 CDA $576 \times 384$ CCD sensor with a full well capacity of 350,000 electrons, a dark current of 8 electrons $\mathrm{s}^{-1}$, and an electronic noise level of 6 electrons; (iii) a 14-bit $50 \mathrm{KHz}$ double correlated analog-to-digital converter; and (iv) a camera controller and image storage and display unit coupled to a central DEC MicroVaxII processing system. Our fluorescence microscope (Zeiss Universal) has been modified by the addition of automated excitation and emission filter selection. In addition, mechanical choppers (Marriott, Clegg, Arndt-Jovin, and Jovin, in preparation) have been placed in the optical paths for excitation and emission in order to provide the capability for measurements of delayed luminescence.

\subsection{Laser Scanning Microscopes}

In the conventional fluorescence microscope, the entire field is illuminated. The resulting emission is either observed visually or photographed, or imaged electronically with a 2-D detector (camera). Scanning microscopes operate on the principle of image formation by illumination and detection on a point-by-point or line-by-line basis. Such systems are of three types: (i) stage or object scanning, (ii) excitation beam scanning, and (iii) detector scanning. The epi-illumination beam scanning system currently used in our laboratory is a Zeiss confocal laser scanning microscope (CLSM) equipped with a tandem galvanometer system for effecting the $x, y$ scan and a stepping motor with $0.1 \mu \mathrm{m}$ resolution for incrementation along the $z$-axis. An accessory UV laser source and external detector have been added to the basic instrument [5]. In the confocal, luminescence mode of operation, emission from the object is collected through a limiting aperture in front of the detector (photomultiplier), thereby generating a confocal image.

In a recent review [3], we have extensively discussed the principles of confocal laser scanning microscopy, according to which the detected intensity originating from the object decays more rapidly in the lateral and axial directions than in the case of the conventional, non-confocal configuration. This feature leads to the following significant improvements in performance: (i) increased lateral resolution, up to a factor of 1.3; (ii) increased axial resolution; (iii) apodization, i.e. attenuation of the side lobes in the focal plane intensity distribution; (iv) suppression of glare and scattering; and (v) suppression of out-of-focus contributions, the feature of greatest importance in the confocal microscope since it enables high quality optical sectioning and, as a consequence, the examination of thick specimens.

\section{Photophysical and Experimental Principles 3.1. Fluorescence Resonance Energy Transfer (FRET) Microscopy}

Recent advances in the technology of light microscopy and data processing have stimulated applications concerned not merely with the determination of position and mass, but also of the state of association, the environment, and dynamic properties of molecules $[2,3,6]$.

One such technique is based on the resonance non-radiative transfer of energy from one excited emission dipole (the donor) to an appropriate absorption dipole (the acceptor), the formalism of which is attributable to Förster $[7,8]$. The efficiency of fluorescence resonance energy transfer (FRET) varies with the 6th power of the distance between donor and acceptor and, therefore, provides a very sensitive, albeit highly non-linear, spectroscopic "ruler" for estimating molecular associations. The following conditions determine whether FRET can compete with other processes deactivating the excited state (Figs. 2 and 3). (i) The donor must be fluorescent. (ii) There must exist a significant overlap between the emission spectrum of the donor and the absorption spectrum of the acceptor. (iii) The distance separating the donor and acceptor must lie within certain limits. (iv) The mutual orientations of the donor and acceptor (i.e., between their respective emission and absorption transition moments) must be favorable. The existence of FRET is manifested

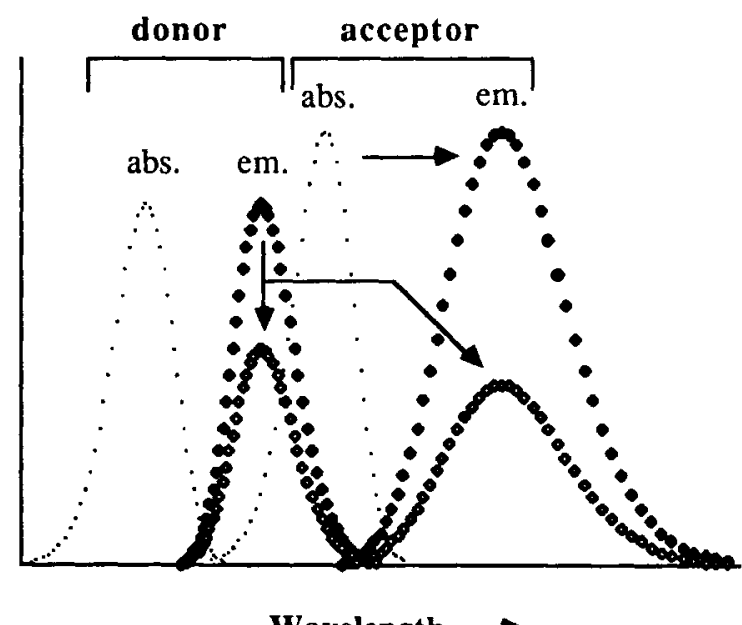

Fig. 2

Spectral manifestations of fluorescence resonance energy transfer (FRET). Absorption spectra of donor and acceptor molecules $(\cdots \cdots)$. Emission from direct excitation shown as spectra $(\bullet)$. When donor and acceptor are in close proximity, excitation of the donor results in quenched emission (vertical arrow toward curve with $\diamond$ symbols). If the acceptor is fluorescent, the chromophores emit with their characteristic (red-shifted) spectrum (inclined arrow toward curve with $\diamond$ symbols). From Ref. [11]

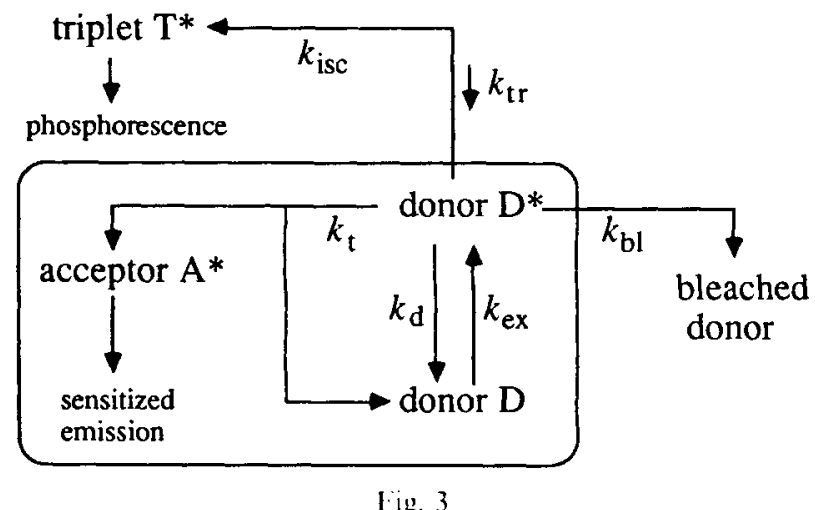

Photophysical scheme for the combined processes of fluorescence energy transfer, donor photobleaching, and delayed luminescence. Rate constants: $k_{\text {ex }}$, donor excitation; $k_{\mathrm{d}}$, decay including $k_{\mathrm{f}}$ for the radiative (fluorescence) pathway; $k_{1}$, nonradiative energy transfer; $k_{\mathrm{bl}}$, irreversible photobleaching; $k_{\text {isc }}$, intersystem crossing to the triplet excited state; $k_{\mathrm{tr}}$, thermal reactivation resulting in delayed fluorescence. From Ref. [3] 
in various ways (Fig. 2). The donor is (partially) quenched with an efficiency designated $E$; that is, the donor emission quantum yield $Q$ is reduced to $Q(1-E)$. The fluorescence lifetime $\tau$ of the donor decreases by the same factor (potentially leading to a corresponding increase in the steady-state emission polarization). If the acceptor is fluorescent, its emission shows an increase in intensity (is sensitized) and a decrease in polarization. A final, previously unappreciated feature discussed further below, is a decrease in the rate of photobleaching of the donor. Thus, the energy transfer efficiency $E$ can be expressed as

$$
E=1-\tau^{\prime} / \tau=1-Q^{\prime} / Q=1-\tau_{\mathrm{bl}} / \tau_{\mathrm{bl}}^{\prime}
$$

in which $\tau^{\prime}$ is the lifetime, $Q^{\prime}$ is the quantum yield, and $\tau_{\mathrm{b}}^{\prime}$ is the photobleaching time of the donor in the double labeled system. The unprimed symbols correspond to the donor alone.

The measurement of FRET in the microscope provides the enormous advantage of spatial resolution over the more conventional determinations carried out in solution, suspension, or in flow cytometry systems. One can assess the immediate environment and interactions of molecules within defined intracellular compartments and organelles, and on cellular surfaces. There are further experimental benefits. In the microscope it is possible to determine and correct for background luminescence and scattering, exclude dead damaged, or otherwise inappropriate cells or structures, reaccess selected regions with different complementary measurements by using combinations of staining and labeling procedures, and examine attached cells for characteristics which cannot be reproduced in suspension.

Although FRET microscope images of cells have been recorded previously by photographic means $[9,10]$ quantitative measurements have been hindered by several problems: (i) a lack of registration of the donor, acceptor, and sensitized emission images, the correction of which is aggravated in the presence of significant cnergy transfer, i.e. extensive donor quenching; (ii) broad distributions and low precision of the calculated energy transfer in the region of low transfer efficiency, particularly if donor emission and direct excitation of the acceptor contribute significantly to the detected signal; (iii) the requirement for determining spectral overlap factors from independent images or solutions; and (iv) the necessity to determine an instrument factor to compensate for differences in absorption cross-section, excitation intensities, and detector efficiencies at the donor and acceptor wavelengths.

The method illustrated in this report is based upon the systematic, total photobleaching of the donor, and is described in detail elsewhere [3,11]. Photobleaching FRET Digital Imaging Microscopy (pbFRET-DIM) exploits the fact that the time-integrated fluorescence emission obtained upon complete photobleaching of a fluorescent molecule is independent of (i) changes in quantum yield accompanying excited state deactivation mechanisms such as resonance energy transfer, and (ii) the particular choice of illumination intensity and duration (Fig. 3, $[3,11]$ ). The Photometrics CCD cam-

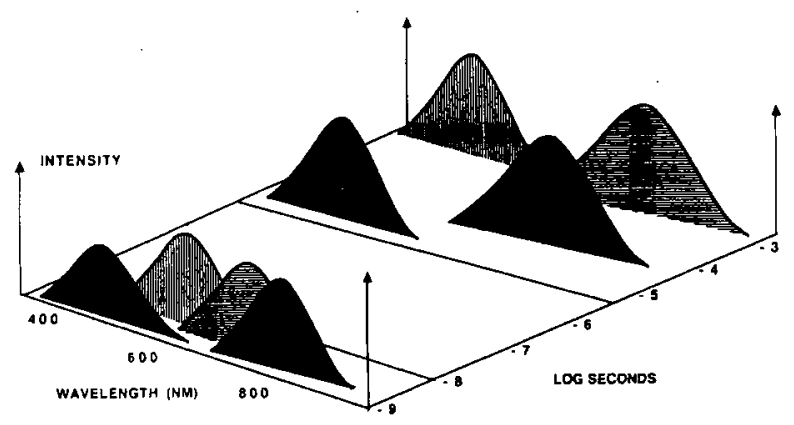

Fig. 4

Spectral-temporal dispersion of huorescence and delayed emission Four spectrally overlapping fluorescence emission spectra are seen to be better separated in the time domain corresponding to delayed emission era system is used to acquire the images, which are then processed by computer in order to establish the energy transfer efficiency on a pixel-by-pixel basis.

The experimental procedure is simple. From a sample labeled with both acceptor and donor fluorophores, one records an image of donor fluorescence with low-level illumination, i.e. conditions minimizing photobleaching. The excitation intensity is then increased and the emission of the donor fluorophore during complete photobleaching is integrated on the CCD sensor. The ratio of the two images yields an image proportional to the quantum yield of the quenched donor. The same determination performed on samples containing only the donor molecules, i.e., in the absence of FRET, provides the proportionality constant required to calculate directly the energy transfer efficiency $E$ (i) for each image point $i$ of interest.

Similar expressions involving the photobleaching rate $1 / \tau_{\mathrm{bl}}(i)$ can also be used to derive $E$ (i) [11]. Thus, from a series of images taken during photobleaching one obtains the rate constant(s) for photobleaching at each pixel position.

\subsection{Delayed Luminescence}

(Fluorescence \& Phosphorescence) Microscopy

A serious problem in quantitative FDIM applied to cellular macromolecules present in low numbers is the contribution from intrinsic cellular autofluorescence and from glare and scattering within the microscope. The use of fluorophores with large Stokes shifts reduces the problem but does not eliminate it. However, one can achieve the required selectivity by exploiting the temporal distribution of luminescence (Fig. 4). Lifetimes of prompt fluorescence have been determined with microscope systems but generally without significant attempts at imaging. We have recently made measurements of long-lived delayed fluorescence and phosphorescence by exploiting the unique advantages of the CCD camera, i.e. with high spatial resolution and sensitivity. This has been achieved with a combination of periodic pulsed excitation and gated detection. A mechanical chopper (or acousto-optic modulator) periodically interrupts the output of a c.w. laser or lamp source, and a second phase-locked chopper in front of the CCD camera is adjusted so as to pass the desired form of luminescence (Marriott, Clegg, ArndtJovin, and Jovin, in preparation). By integration within a time window of adjustable phase and width, one can record either the prompt fluorescence or the delayed luminescence. In the latter case, very efficient rejection of interference from the much more intense prompt fluorescence is achieved. Lifetimes are evaluated for each pixel of interest from a sequence of images generated by systematically changing either the time delay between excitation and detection or the chopping frequency.

\section{Results and Discussion}

We describe briefly applications of the three LDIM techniques discussed above to the study of particular questions involving DNA distribution and metabolism. The pbFRETDIM method was used in studies of polytene chromosomes stained with specific DNA dyes $[11,12]$. The donor was bisbenzimidazole 33342 (BBI-342), an A-T specific drug which binds in the minor groove of DNA, and the acceptor was the intercalator ethidium bromide. Chromosome preparations were stained with donor $(10 \mu \mathrm{M})$ alone or in combination with the acceptor $(50 \mu \mathrm{M})$. Images were taken of the same chromosomes at successive time intervals during photobleaching using full intensity excitation with a mercury arc lamp. All records were corrected for bias and dark current, field inhomogeneity of the excitation source, and background. In Fig. 5 are plotted the log of the mean of the intensity of chromosome 1 determined at intervals during photobleaching from preparations stained with donor alone or with both donor and acceptor. The bleaching time for 


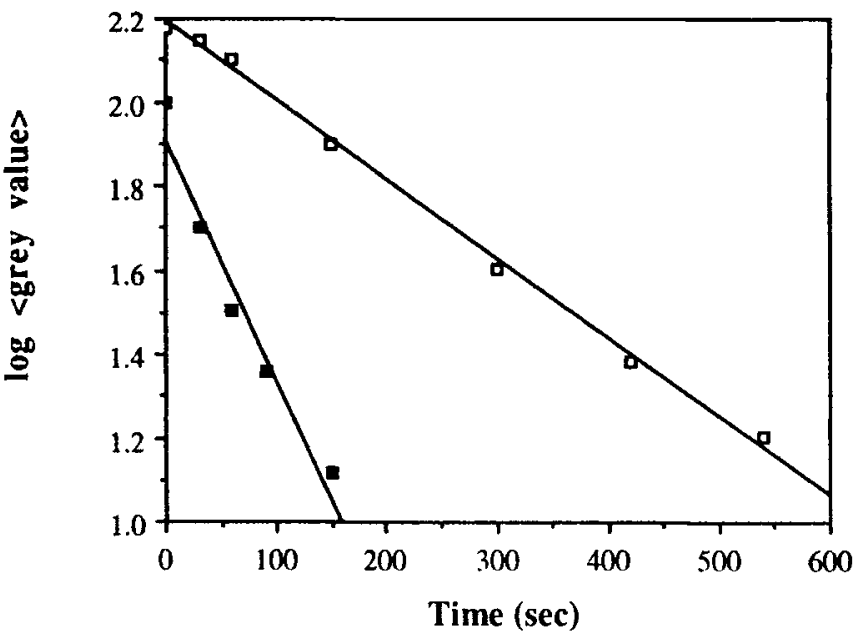

Fig. 5

Photobleaching kinetics of DNA dyes bound to polytene chromosomes. The $\log _{10}$ of the mean intensity of successive images acquired during photobleaching is plotted versus time for single and doubly labeled chromosomes. The bleaching time $\tau_{b l}$ may be derived from the indicated linear regressions assuming a first-order decay process: for the single labeled samples $(\boldsymbol{E}), \tau_{\mathrm{b}}=75 \mathrm{~s}$; and for the double labeled samples $(\square), \tau_{b}^{\prime}=230 \mathrm{~s}$. From Ref. [11]

the donor alone is $75 \mathrm{~s}$ which increases in the presence of acceptor to $230 \mathrm{~s}$, corresponding to an energy transfer efficiency of $67 \%$ calculated according to Eq. (1) (see Refs. [11] and [12] for further details). These results show that the intercalator is in close proximity to the BBI-342 binding sites on the chromosome. Further studies with a variety of sequence specific drugs as well as non-fluorescent acceptors are in progress in order to probe local interactions at chromosome loci with single pixel resolution. The method is also being applied to the determination of the spatial relationship between DNA replication sites and the nuclear envelope using a monoclonal antibody against BrdU as the donor, and a monoclonal antibody to lamin, a nuclear matrix protein, as the acceptor. FRET microscopy is being applied in other cell biological investigations, for example of interactions between specific cell surface receptors and antigens.

We have investigated the binding of the metachromatic dye acridine orange in living and fixed cells by measurements in the time domain with the technique of delayed Luminescence Digital Imaging Microscopy (Marriott, Clegg, Arndt-Jovin, and Jovin, in preparation). In both polytene and diploid cells, the dye displays a long $(0.4 \mathrm{~ms})$ delayed fluorescence and phosphorescence lifetime when bound to cellular DNA in the absence of oxygen. Delayed luminescence images have intensities of $\sim 0.1 \%$ relative to the corresponding fluorescence images (at 0 phase shift). The components of scattering and diffuse fluorescence from unbound dye in solution are completely blocked by the mechanical chopper mounted in front of the CCD camera. In the present instrument, the measurements of delayed luminescence are limited to lifetimes longer than $50 \mu \mathrm{s}$.

In addition, we have exploited the potentiation of intersystem crossing to the triplet state by heavy atom substituents [13]. In the experiment shown below, cells were grown in the presence of the thymidine analog BrdU for 12 hours, during which time most cells underwent a round of replication leading to the incorporation of $\mathrm{BrdU}$ into the DNA. In Fig. 6, a living rat basophilic leukemia cell is shown after exposure to BrdU and staining in vivo with acridine orange. The fluorescence image (panel $\mathrm{A}$ ) is dominated by emission from cytoplasmic lysosomes, whereas only the nucleus is apparent in the delayed luminescence image of the same cell (panel B). The intensity of the delayed luminescence image is $5 \%$ of the maximum in the fluorescence image, a 50-fold enhancement over the much weaker phosphorescence signals originating from other regions of the cell in which the external heavy atom effect is not operative. We have detected living replicating cells in culture by this method using shorter pulses of BrdU incorporation (Marriott, Clegg, Arndt-Jovin, and Jovin, in preparation). Such experiments demonstrate that specific macromolecules and structures can be studied in living cells with this microscope technique. The interfering signals contributed by cellular autofluorescence and by emission of the unbound probe are effectively suppressed.

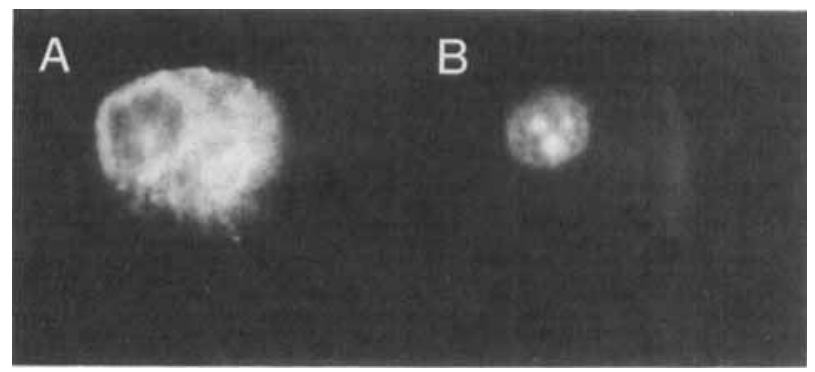

Fig. 6

Fluorescence and delayed luminescence images of living rat basophilic leukemia cells stained with acridine orange after $12 \mathrm{hr}$. replication in bromodeoxyuridine. Excitation was at $436 \mathrm{~nm}$ with a 100 watt $\mathrm{Hg}$ arc lamp chopped at a frequency of $534 \mathrm{~Hz}$. Emission was collected above $515 \mathrm{~nm}$ by imaging with the Photometrics CCD camera (see text for details). Objective, planapo $63 \times$ oil immersion, NA 1.3. (A) Fluorescence image acquired with an integration time of $1 \mathrm{~s}$ with the emission chopper in a free running mode. (B) Delayed luminescence acquired for $20 \mathrm{~s}$ integration time with the ernission chopper set out of phase with the excitation chopper

Many studies in cell biology involve the examination of intact structures and organisms. As a example one can cite pattern formation and determination in early embryogenesis, characterized by inhibition or stimulation of cell growth and differentiation over substantial distances. Embryos constitute optically thick specimens. Until recently, the spatial distribution of internal components were accessible only through physical thin-sectioning and observation with conventional light microscopes and electron microscopes. These procedures often distort the essential structural features of interest and, of course, are incompatible with measurements in the living state. Many embryos are translucent; light can penetrate through most, if not all of the cellular mass. Thus, one can perform optical sectioning on intact specimens using the confocal laser scanning microscope. In addition, certain probes penetrate the living embryo or can be microinjected, allowing the quantitative measurement of a number of macromolecules and structures in vivo. Alternatively, a "snapshot" can be obtained by rapid fixation of the entire embryo and staining of the whole mount preparations with specific 
antibodies, nucleic acid hybridization probes, or other luminescent molecules.

The early stages of embryogenesis are characterized by rapid replication and transcription of the genome. Under these conditions, the topological state of chromatin is regulated by the action of specific enzymes which can change the local or global superhelix density of the DNA. We have studied the distribution of one of these enzymes, topoisomerase II, during Drosophila development. Early Drosophila embryos, about $100 \mu$ thick, were fixed as whole mount preparations and stained with a DNA specific dye, mithramycin, as well as an antibody against topoisomerase II. Optical sections of the embryos were imaged with the CLSM using two different wavelengths of laser excitation. We assessed the degree of colocalization from the superimposed intensity distributions corresponding to the two probes. The two images from a single optical section (Fig. 7) reveal that about $50 \%$ of the topoisomerase II is localized on the chromatin during interphase.

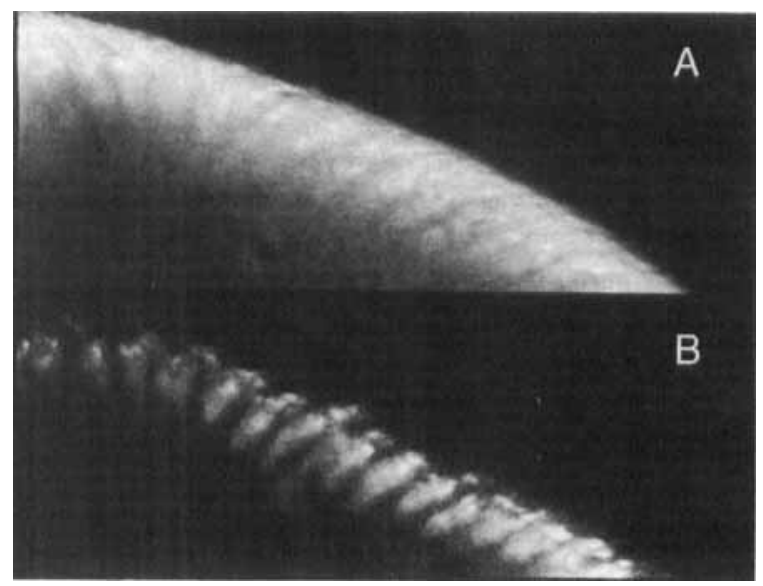

Fig. 7

Optical sectioning by confocal laser scanning microscopy. A single optical section through a whole mount Drosophila embryo stained with (A) anti-topoisomerase II, and (B) mithramycin as a DNA marker. Excitation: for mithramycin, argon ion laser at $458 \mathrm{~nm}$; for indirect rhodamine labeled antibody, argon ion laser at $514 \mathrm{~nm}$

\section{Conclusions}

The measurements presented above demonstrate that new photophysical phenomena measured with digital imaging microscopes extend the qualitative and quantitative nature of the information that can be derived from cell biological applications. It can be anticipated [3] that further developments will proceed rapidly, particularly in the case of combined technologies offering the prospect of superresolution.
We have stressed the utility of temporal resolution achieved with pulsed illumination. Alternatively, excitation can be achieved with continuously modulated light sources, in which case the luminescence signals display a phase shift and modulation depth related to the lifetimes of the probe(s) [14]. By measurement at a specific relative phase corresponding to a given modulation frequency, one can achieve selectivity for a component of interest and rejection of unwanted background. An additional feature, not yet exploited in the microscope, is that of differential polarization.

The confocal laser scanning microscope equipped with point-by-point illumination and detection is also ideally suited for measurements of temporally resolved excitation and emission processes. However, more versatile detection systems are required than those presently available on commercial CLSM instruments. That is, one could incorporate pulsed or modulated excitation, gated or phase-sensitive detection, multispectral sampling, and photon counting. In this sense, the CSLM can be regarded as a spectroscopic, photometric device, equipped with laser excitation and electrooptic detection, and differing from the conventional "cuvette" instrument primarily in that it samples up to a million spatially resolved points in rapid succession.

\section{References}

[1] S. Inoué, "Video Microscopy", Plenum, New York 1986.

[2] D. J. Arndt-Jovin, M. Robert-Nicoud, S. J. Kaufmann, and T. M. Jovin, Science 230, 247 (1985).

[3] T. M. Jovin and D. J. Arndt-Jovin, Annu. Rev. Biophys. Chem. 18, 271 (1989).

[4] J. R. Janesick (Ed.), Opt. Eng. 26 (Nos. 8-10), 685-1083 (1987).

[5] M. Robert-Nicoud, D. J. Arndt-Jovin, T. Schormann, and T. M. Jovin, Eur. J. Cell Biol., in press.

[6] D. L. Taylor, A. S. Waggoner, R. F. Murphy, F. Lanni, and R. R. Birge (Eds.), "Applications of Fluorescence in the Biomedical Sciences", Alan Liss, New York 1986.

[7] T. Förster, Ann. Phys., Leipzig 2, 55 (1948).

[8] T. Förster, "Fluoreszenz Organischer Verbindungen", Vandenhoeck \& Ruprecht, Göttingen 1951.

[9] S. S. Chan, D. J. Arndt-Jovin, and T. M. Jovin, J. Histochem. Cytochem. 27, 56 (1979).

[10] P. S. Uster and R. E. Pagano, J. Cell Biol. 103, 1221 (1986).

[11] T. M. Jovin and D. J. Arndt-Jovin, in: "Microspectrofluorometry of Single Living Cells", eds. E. Kohen, J. S. Ploem, and J. G. Hirschberg, Academic Press, Orlando, FL, in press.

[12] D. J. Arndt-Jovin and T. M. Jovin, Methods Cell Biol. 29, 417 (1988).

[13] A. F. Corin and T. M. Jovin, Biochemistry 25, 3995 (1986).

[14] E. Gratton, D. M. Jameson, and R. D. Hall, Annu. Rev. Biophys. Biophys. Chem. 13, 105 (1984).

Presented at the Discussion Meeting of the Deutsche Bunsen-Gesellschaft für Physikalische Chemie "Laser in Life Sciences", University of Heidelberg, August 29th to September 1st, 1988 